International Mathematical Forum, Vol. 9, 2014, no. 7, 317 - 324

HIKARI Ltd, www.m-hikari.com

http://dx.doi.org/10.12988/imf.2014.4113

\title{
Uniqueness of Meromorphic Functions of Differential Polynomials Sharing a Small Function IM
}

\author{
Xinhua Shi \\ College of Science \\ Civil Aviation University of China \\ Tianjin 300300, China
}

Copyright (c) 2014 Xinhua Shi. This is an open access article distributed under the Creative Commons Attribution License, which permits unrestricted use, distribution, and reproduction in any medium, provided the original work is properly cited.

\begin{abstract}
In this paper, we shall study the uniqueness problems of meromorphic functions of differential polynomials sharing a small function and $\infty$ IM. Our results improve or generalize many previous results on value sharing of meromorphic functions.
\end{abstract}

Mathematics Subject Classification: 30D30, 30D35

Keywords: differential polynomial, uniqueness, meromorphic function, shared values

\section{Introduction and main results}

Let $f(z)$ and $g(z)$ be two non-constant meromorphic functions defined in the open complex plane $\mathbb{C}$. Let $a$ be a small function with respect to $f$ and $g$. We say that $f(z), g(z)$ share $a(z) \mathrm{CM}$ (counting multiplicities) if $f(z)-a(z), g(z)-a(z)$ have the same zeros with the same multiplicities and we say that $f(z), g(z)$ share $a(z)$ IM (ignoring multiplicities) if we do not consider the multiplicities. We denote by $T(r, f)$ the Nevanlinna characteristic function of the meromorphic function $f$ and by $S(r, f)$ any quantity satisfying $S(r, f)=o(T(r, f))$ as $r \rightarrow \infty$ possibly outside a set of finite linear measure. $N_{k}(r, f)$ denotes the 
truncated counting function bounded by $k$. Moreover, $G C D\left(n_{1}, n_{2}, \cdots, n_{k}\right)$ denotes the greatest common divisor of positive integers $n_{1}, n_{2}, \cdots, n_{k}$.

For the sake of simplicity, let $m$ be a nonnegative integer, $a_{0} \neq 0$, $a_{1}, \cdots, a_{m-1}, a_{m} \neq 0$ be complex constants. Define

$$
P(w)= \begin{cases}a_{m} w^{m}+a_{m-1} w^{m-1}+\cdots+a_{1} w+a_{0}, & >0, \\ a_{0}, & m=0 .\end{cases}
$$

In 2011, Zhang and $\mathrm{Xu}$ [12] considered uniqueness problem on meromorphic functions sharing a small function CM and proved the following result.

Theorem A Let $f$ and $g$ be two non-constant meromorphic functions, $a(z)(\not \equiv$ $0, \infty)$ be a small function with respect to $f$. Let $n, k$, and $m$ be three positive integers with $n>3 k+m+8$ and $P(w)$ be defined as in Theorem $G$. If $\left[f^{n} P(f)\right]^{(k)}$ and $\left[g^{n} P(g)\right]^{(k)}$ share a $C M$, then

(I) when $P(w)=a_{m} w^{m}+a_{m-1} w^{m-1}+\cdots+a_{1} w+a_{0}$, one of the following three cases holds:

(I1) $f(z) \equiv t g(z)$ for a constant $t$ such that $t^{d}=1$, where $d=G C D(n+$ $m, \cdots, n+m-i, \cdots, n), a_{m-i} \neq 0$ for some $i=0,1, \cdots, m$,

(I2) $f$ and $g$ satisfy the algebraic equation $R(f, g) \equiv 0$, where $R\left(\omega_{1}, \omega_{2}\right)=$ $\omega_{1}^{n}\left(a_{m} \omega_{1}^{m}+a_{m-1} \omega_{1}^{m-1}+\cdots+a_{0}\right)-\omega_{2}^{n}\left(a_{m} \omega_{2}^{m}+a_{m-1} \omega_{2}^{m-1}+\cdots+a_{0}\right)$,

(I3) $\left[f^{n} P(f)\right]^{(k)}\left[g^{n} P(g)\right]^{(k)}=a^{2}$;

(II) when $P(w) \equiv a_{0}$, one of the following two cases holds:

(II1) $f \equiv$ tg for a constant $t$ such that $t^{n}=1$,

(II2) $a_{0}^{2}\left[f^{n}\right]^{(k)}\left[g^{n}\right]^{(k)}=a^{2}$.

In 2014, Li, Qiu and Xuan [3] changed CM sharing to IM sharing and got Theorem B Let $f$ and $g$ be two transcendental meromorphic functions, $a(z)(\not \equiv$ $0, \infty)$ be a small function with respect to $f$. Let $n, k$, and $m$ be three positive integers with $n>4 m+9 k+14$ and $P(w)$ be defined as in Theorem $G$. If $\left[f^{n} P(f)\right]^{(k)}$ and $\left[g^{n} P(g)\right]^{(k)}$ share a IM, then

(I) when $P(w)=a_{m} w^{m}+a_{m-1} w^{m-1}+\cdots+a_{1} w+a_{0}$, one of the following three cases holds:

(I1) $f(z) \equiv \operatorname{tg}(z)$ for a constant $t$ such that $t^{d}=1$, where $d=G C D(n+$ $m, \cdots, n+m-i, \cdots, n), a_{m-i} \neq 0$ for some $i=0,1, \cdots, m$,

(I2) $f$ and $g$ satisfy the algebraic equation $R(f, g) \equiv 0$, where $R\left(\omega_{1}, \omega_{2}\right)=$ $\omega_{1}^{n}\left(a_{m} \omega_{1}^{m}+a_{m-1} \omega_{1}^{m-1}+\cdots+a_{0}\right)-\omega_{2}^{n}\left(a_{m} \omega_{2}^{m}+a_{m-1} \omega_{2}^{m-1}+\cdots+a_{0}\right)$,

(I3) $\left[f^{n} P(f)\right]^{(k)}\left[g^{n} P(g)\right]^{(k)}=a^{2}$;

(II) when $P(w) \equiv a_{0}$, one of the following two cases holds:

(II1) $f \equiv t g$ for a constant $t$ such that $t^{n}=1$,

(II2) $a_{0}^{2}\left[f^{n}\right]^{(k)}\left[g^{n}\right]^{(k)}=a^{2}$.

In this paper, we shall further improve Theorem B by reducing the lower 
bound of $n$. We got

Theorem 1.1. Let $f$ and $g$ be two non-constant meromorphic functions, $a(z)(\not \equiv$ $0, \infty)$ be a small function with respect to $f$, which has no common zero or poles with $f$ and $g$. Let $n, k$, and $m$ be three positive integers with $n>4 m+9 k+10$ and $P(w)$ be defined as in Theorem G. If $\left[f^{n} P(f)\right]^{(k)}$ and $\left[g^{n} P(g)\right]^{(k)}$ share a $I M, f$ and $g$ share $\infty I M$, then

(I) when $P(w)=a_{m} w^{m}+a_{m-1} w^{m-1}+\cdots+a_{1} w+a_{0}$, one of the following three cases holds:

(I1) $f(z) \equiv \operatorname{tg}(z)$ for a constant $t$ such that $t^{d}=1$, where $d=G C D(n+$ $m, \cdots, n+m-i, \cdots, n), a_{m-i} \neq 0$ for some $i=0,1, \cdots, m$,

(I2) $f$ and $g$ satisfy the algebraic equation $R(f, g) \equiv 0$, where $R\left(\omega_{1}, \omega_{2}\right)=$ $\omega_{1}^{n}\left(a_{m} \omega_{1}^{m}+a_{m-1} \omega_{1}^{m-1}+\cdots+a_{0}\right)-\omega_{2}^{n}\left(a_{m} \omega_{2}^{m}+a_{m-1} \omega_{2}^{m-1}+\cdots+a_{0}\right)$;

(II) when $P(w) \equiv a_{0}$, one of the following two cases holds:

(II1) $f \equiv$ tg for a constant $t$ such that $t^{n}=1$,

(II2) $a_{0}^{2}\left[f^{n}\right]^{(k)}\left[g^{n}\right]^{(k)}=a^{2}$.

Theorem 1.2. Let $f$ and $g$ be two transcendental meromorphic functions, $p(z)$ be a nonzero polynomial with $\operatorname{deg}(p)=l \leq 5$, which has no common zero with $f$ and $g$. Let $n, k$ and $m$ be three positive integers with $n>4 m+9 k+10$. Let $P(w)=a_{m} w^{m}+a_{m-1} w^{m-1}+\cdots+a_{1} w+a_{0}$ be a nonzero polynomial. If $\left[f^{n} P(f)\right]^{(k)}$ and $\left[g^{n} P(g)\right]^{(k)}$ share $p I M, f$ and $g$ share $\infty$ IM. Then one of the following three cases holds:

(1) $f(z) \equiv t g(z)$ for a constant $t$ such that $t^{d}=1$, where $d=G C D(n+$ $m, \cdots, n+m-i, \cdots, n), a_{m-i} \neq 0$ for some $i=0,1, \cdots, m$;

(2) $f$ and $g$ satisfy the algebraic equation $R(f, g) \equiv 0$, where $R\left(\omega_{1}, \omega_{2}\right)=$ $\omega_{1}^{n}\left(a_{m} \omega_{1}^{m}+a_{m-1} \omega_{1}^{m-1}+\cdots+a_{0}\right)-\omega_{2}^{n}\left(a_{m} \omega_{2}^{m}+a_{m-1} \omega_{2}^{m-1}+\cdots+a_{0}\right)$;

(3) $P(z)$ is reduced to a nonzero monomial, namely, $P(z)=a_{i} z^{i} \not \equiv 0$ for some $i \in\{0,1, \cdots, m\}$; if $p(z)$ is not a constant, then $f=c_{1} e^{c Q(z)}, g=c_{2} e^{-c Q(z)}$, where $Q(z)=\int_{0}^{z} p(z) d z, c_{1}, c_{2}$ and c are constants such that $a_{i}^{2}\left(c_{1} c_{2}\right)^{n+i}[(n+$ i)c] $]^{2}=-1$,

if $p(z)$ is a nonzero constant $b$, then $f=c_{3} e^{c z}, g=c_{4} e^{-c z}$, where $c_{3}, c_{4}$ and $c$ are constants such that $(-1)^{k} a_{i}^{2}\left(c_{3} c_{4}\right)^{n+i}[(n+i) c]^{2 k}=b^{2}$.

\section{Preliminary lemmas}

Let

$$
\begin{aligned}
& H=\left(\frac{F^{\prime \prime}}{F^{\prime}}-\frac{2 F^{\prime}}{F-1}\right)-\left(\frac{G^{\prime \prime}}{G^{\prime}}-\frac{2 G^{\prime}}{G-1}\right), \\
& V=\left(\frac{F^{\prime}}{F-1}-\frac{F^{\prime}}{F}\right)-\left(\frac{G^{\prime}}{G-1}-\frac{G^{\prime}}{G}\right),
\end{aligned}
$$


where $F=\left[f^{n} P(f)\right]^{(k)} / a(z)$ and $G=\left[g^{n} P(g)\right]^{(k)} / a(z)$, both $f$ and $g$ are meromorphic functions, $a(z)(\not \equiv 0, \infty)$ be a small function with respect to both $f$ and $g$, which has no common zero or poles with $f$ and $g$.

Lemma 2.1. [6] Let $f(z)$ be a non-constant meromorphic function and let $a_{0}(z), a_{1}(z), \cdots, a_{n}(z)(\not \equiv 0)$ be small functions with respect to $f$. Then

$$
T\left(r, a_{n} f^{n}+a_{n-1} f^{n-1}+\cdots+a_{0}\right)=n T(r, f)+S(r, f) .
$$

Lemma 2.2. [4] Let $f(z)$ be a non-constant meromorphic function, $s, k$ be two positive integers. Then

$$
\begin{gathered}
N_{s}\left(r, \frac{1}{f^{(k)}}\right) \leq T\left(r, f^{(k)}\right)-T(r, f)+N_{s+k}\left(r, \frac{1}{f}\right)+S(r, f), \\
N_{s}\left(r, \frac{1}{f^{(k)}}\right) \leq k \bar{N}(r, f)+N_{s+k}\left(r, \frac{1}{f}\right)+S(r, f) .
\end{gathered}
$$

Lemma 2.3. [2, 8, 9] Let $f(z)$ be a non-constant meromorphic function, and let $k$ be a positive integer. Suppose that $f^{(k)} \not \equiv 0$, then

$$
N\left(r, \frac{1}{f^{(k)}}\right) \leq N\left(r, \frac{1}{f}\right)+k \bar{N}(r, f)+S(r, f) .
$$

By using the similar method to Banerjee [1, Lemma 2.14], we can prove the following Lemma.

Lemma 2.4. Let $F, G$ and $H$ be defined as in (2.1). If $F$ and $G$ share 1 IM and $\infty I M$, and $H \not \equiv 0$, then $F \not \equiv G$, and

$$
\begin{aligned}
T(r, F) & \leq N_{2}(r, 1 / F)+N_{2}(r, 1 / G)+2 \bar{N}(r, 1 / F)+\bar{N}(r, 1 / G) \\
& +6 \bar{N}(r, F)+S(r, F)+S(r, G),
\end{aligned}
$$

the same inequality holding for $T(r, G)$.

Lemma 2.5. [10] Let $F, G$ and $V$ be defined as in (2.2). If $F$ and $G$ share $\infty I M$, and $V \equiv 0$, then $F \equiv G$.

Lemma 2.6. [11] If $F$ and $G$ share 1 IM, then

$$
\bar{N}_{L}\left(r, \frac{1}{F-1}\right) \leq \bar{N}(r, 1 / F)+\bar{N}(r, F)+S(r, F)+S(r, G),
$$

Lemma 2.7. Let $f, g$ be two non-constant meromorphic functions, $F, G$ and $V$ be defined as in (2.2), $P(w)$ is defined as in (1.1), $n>0, k>0$ and $m \geq 0$ are three integers. If $V \not \equiv 0, F$ and $G$ share 1 and $\infty I M$, then

$$
\begin{aligned}
& (n+m+k-3) \bar{N}(r, f)=(n+m+k-3) \bar{N}(r, g) \\
\leq & 2 \bar{N}(r, 1 / F)+2 \bar{N}(r, 1 / G)+S(r, f)+S(r, g) .
\end{aligned}
$$


Proof. Since $V \not \equiv 0, f$ and $g$ share $\infty$ IM, suppose that $z_{0}$ is a pole of $f$ with multiplicity $p$, a pole of $g$ with multiplicity $q$, then $z_{0}$ is a pole of $F$ with multiplicity $(n+m) p+k$, a pole of $G$ with multiplicity $(n+m) q+k$, thus $z_{0}$ is a zero of $\frac{F^{\prime}}{F-1}-\frac{F^{\prime}}{F}=\frac{F^{\prime}}{F(F-1)}$ with multiplicity $(n+m) p+k-1(\geq n+m+k-1)$, and $z_{0}$ is a zero of $\frac{G^{\prime}}{G-1}-\frac{G^{\prime}}{G}=\frac{G^{\prime}}{G(G-1)}$ with multiplicity $(n+m) q+k-1(\geq$ $n+m+k-1)$, hence $z_{0}$ is a zero of $V$ with multiplicity at least $n+m+k-1$. So

$$
(n+m+k-1) \bar{N}(r, f)=(n+m+k-1) \bar{N}(r, g) \leq N\left(r, \frac{1}{V}\right)
$$

By the logarithmic derivative lemma, we have $m(r, V)=S(r, f)+S(r, g)$. Note that $F$ and $G$ share 1 IM, by Lemma 2.6 , so we have

$$
\begin{aligned}
N\left(r, \frac{1}{V}\right) \leq & T(r, V)=m(r, V)+N(r, V) \\
\leq & \bar{N}(r, 1 / F)+\bar{N}(r, 1 / G)+\bar{N}_{L}\left(r, \frac{1}{F-1}\right) \\
& +\bar{N}_{L}\left(r, \frac{1}{G-1}\right)+S(r, f)+S(r, g) \\
\leq & 2 \bar{N}(r, 1 / F)+2 \bar{N}(r, 1 / G)+2 \bar{N}(r, f) \\
& +S(r, f)+S(r, g) .
\end{aligned}
$$

From (2.4) and (2.5) we get (2.3). This proves Lemma 2.6.

By the same reason as in Lemma 5 of [5], we obtain the following lemma.

Lemma 2.8. Let $f$ and $g$ be two non-constant meromorphic functions. Let $P(w)$ be defined as in (1.1), and $n>0, k>0$, and $m \geq 0$ be three integers with $n>2 k+m+1$. If $\left[f^{n} P(f)\right]^{(k)}=\left[g^{n} P(g)\right]^{(k)}$, then $f^{n} P(f)=g^{n} P(g)$.

By using the similar method to Lemma 2.9 of [12], we have

Lemma 2.9. Let $f$ and $g$ be non-constant meromorphic functions, $n, k$ be two positive integers with $n>k+2$, and let $P(w)$ be defined as in (1.1), $a(z)(\not \equiv 0, \infty)$ be a small function with respect to $f$, which has no common zero or poles with $f$ and $g$. If $\left[f^{n} P(f)\right]^{(k)}\left[g^{n} P(g)\right]^{(k)}=a^{2}$, $f$ and $g$ share $\infty$ IM, then $P(w)$ is reduced to a nonzero monomial.

\section{Proof of Theorem 1.1}

Let $F, G, H$ and $V$ be defined in (2.1) and (2.2). $F_{1}=f^{n} P(f), G_{1}=g^{n} P(g)$, then $F$ and $G$ share 1 and $\infty$ IM.

Suppose that $H \not \equiv 0$, then $F \not \equiv G$, and $V \not \equiv 0$. 
Case 1. $m>0$. By Lemma 2.4 we have

$$
\begin{aligned}
T(r, F) & \leq N_{2}(r, 1 / F)+N_{2}(r, 1 / G)+2 \bar{N}(r, 1 / F)+\bar{N}(r, 1 / G) \\
& +6 \bar{N}(r, F)+S(r, F)+S(r, G),
\end{aligned}
$$

By Lemma 2.2 with $s=2$, we obtain

$$
T\left(r, F_{1}\right) \leq T(r, F)-N_{2}(r, 1 / F)+N_{k+2}\left(r, 1 / F_{1}\right)+S(r, F),
$$

and

$$
N_{2}(r, 1 / G) \leq N_{k+2}\left(r, 1 / G_{1}\right)+k \bar{N}(r, G)+S(r, G)
$$

Combining (3.1)-(3.3) gives

$$
\begin{aligned}
T\left(r, F_{1}\right) \leq & N_{k+2}\left(r, 1 / F_{1}\right)+N_{k+2}\left(r, 1 / G_{1}\right)+(4 k+6) \bar{N}(r, f) \\
& +2 N_{k+1}\left(r, 1 / F_{1}\right)+N_{k+1}\left(r, 1 / G_{1}\right)+S(r, f)+S(r, g) \\
\leq & (3 k+4) \bar{N}(r, 1 / f)+3 N(r, 1 / P(f))+(2 k+3) \bar{N}(r, 1 / g) \\
& +2 N(r, 1 / P(g))+(4 k+6) \bar{N}(r, f)+S(r, f)+S(r, g) .
\end{aligned}
$$

It follows from Lemma 2.1 and the above inequality that

$$
\begin{aligned}
(n+m) T(r, f) & \leq(3 k+4) \bar{N}(r, 1 / f)+3 N(r, 1 / P(f))+(2 k+3) \bar{N}(r, 1 / g) \\
& +2 N(r, 1 / P(g))+(4 k+6) \bar{N}(r, f)+S(r, f)+S(r, g) .(3.4)
\end{aligned}
$$

Similarly we have

$$
\begin{aligned}
(n+m) T(r, g) & \leq(3 k+4) \bar{N}(r, 1 / g)+3 N(r, 1 / P(g))+(2 k+3) \bar{N}(r, 1 / f) \\
& +2 N(r, 1 / P(f))+(4 k+6) \bar{N}(r, f)+S(r, f)+S(r, g)(3.5)
\end{aligned}
$$

From (3.4) and (3.5) we deduce that

$$
\begin{aligned}
(n-5 k-4 m-7)(T(r, f)+T(r, g)) & \leq(4 k+6) \bar{N}(r, f) \\
& +S(r, f)+S(r, g) .
\end{aligned}
$$

Note that $V \not \equiv 0$ and we get (2.3). By Lemma 2.2 with $s=1$, we obtain

$$
\begin{aligned}
\bar{N}(r, 1 / F) \leq & N_{k+1}\left(r, 1 / F_{1}\right)+k \bar{N}(r, f)+S(r, f) \\
\leq & (k+1) \bar{N}(r, 1 / f)+N(r, 1 / P(f)) \\
& +k \bar{N}(r, f)+S(r, f) .
\end{aligned}
$$

and

$$
\begin{aligned}
\bar{N}(r, 1 / G) \leq & N_{k+1}\left(r, 1 / G_{1}\right)+k \bar{N}(r, g)+S(r, g) \\
\leq & (k+1) \bar{N}(r, 1 / g)+N(r, 1 / P(g)) \\
& +k \bar{N}(r, g)+S(r, g) .
\end{aligned}
$$


From (2.3), (3.7) and (3.8) we get

$$
\begin{aligned}
(n+m-3 k-3) \bar{N}(r, f) \leq & 2(k+m+1)(T(r, f)+T(r, g)) \\
& +S(r, f)+S(r, g) .
\end{aligned}
$$

Combining (3.6)-(3.9) gives

$$
\begin{aligned}
& {[(n-5 k-4 m-7)(n+m-3 k-3)-4(4 k+6)(k+m+1)]} \\
& (T(r, f)+T(r, g)) \leq S(r, f)+S(r, g)
\end{aligned}
$$

which is a contradiction since $n>4 m+9 k+10$.

Thus $H \equiv 0$. Similar to the proof of [7, Lemma 3], we obtain

(i) $\left[f^{n} P(f)\right]^{(k)}\left[g^{n} P(g)\right]^{(k)}=a^{2}$, or

(ii) $\left[f^{n} P(f)\right]^{(k)} \equiv\left[g^{n} P(g)\right]^{(k)}$.

By Lemma 2.9, the case of $(i)$ is impossible. By Lemma 2.8, we get $f^{n} P(f) \equiv g^{n} P(g)$ from $(i i)$.

Case 2. $m=0$. Similar to the proof of Case 1 , we get

$$
\begin{aligned}
& {[(n-5 k-7)(n-3 k-3)-4(4 k+6)(k+1)](T(r, f)+T(r, g))(3 .} \\
& \leq S(r, f)+S(r, g)
\end{aligned}
$$

which is a contradiction since $n>9 k+10$.

Thus $H \equiv 0$. and we have

(iii) $a_{0}^{2}\left[f^{n}\right]^{(k)}\left[g^{n}\right]^{(k)}=a^{2}$, or

(iv) $\left[f^{n}\right]^{(k)} \equiv\left[g^{n}\right]^{(k)}$.

For $(i v)$, By Lemma 2.8, we get $f(z) \equiv \operatorname{tg}(z)$ for a constant $t$ such that $t^{n}=1$.

This completes the proof of Theorem 1.1.

\section{Proof of Theorem 1.2}

By the similar method to the proof of Theorem 1.1, we only need to consider

$$
\left[f^{n} P(f)\right]^{(k)}\left[g^{n} P(g)\right]^{(k)}=p^{2}
$$

Similar to the proof of Theorem 1.3 of [5], we obtain the results. 


\section{References}

[1] A. Banerjee, Meromorphic functions sharing one value, Int. J. Math. Math. Sci. 22 (2005) 3587-3598.

[2] W.K. Hayman, Meromorphic Functions, Clarendon Press, Oxford, 1964.

[3] R.J. Li, L. Qiu, Z.X. Xuan, Uniqueness of meromorphic functions sharing one small function, Journal of Computational and Applied Mathematics, 263 (2014), 225C-235.

[4] W.C. Lin and H.X. Yi, Uniqueness theorems for meromorphic

[5] J.F. Xu, F. Lü and H.X. Yi, Fixed-points and uniqueness of meromorphic functions, Comput. Math. Appl. 59 (2010), 9-17.

[6] C.C. Yang, On deficiencies of differential polynomials II, Math. Z. 125 (1972), 107-112.

[7] C.C. Yang and X.H. Hua, Uniqueness and value-sharing of meromorphic functions, Ann. Acad. Sci. Fenn. Math. 22 (1997), 395-406.

[8] C.C. Yang and H.X. Yi, Uniqueness theory of meromorphic functions, Kluwer Acad. Publ. Dordrecht, 2003.

[9] L. Yang, Value Distribution Theory, Springer-Verlag, Berlin, 1993.

[10] H.X. Yi, Meromorphic functions that share three sets, Kodai Math. J. 20 (1997), 22-32.

[11] H.X. Yi, Meromorphic functions that share one or two values. II, KodaiMath. J. 22 (1999), 264-272.

[12] X.B. Zhang, J.F. Xu, Uniqueness of meromorphic functions sharing a small function and its applications. Comput. Math. Appl. 61 (2011), 722730 .

Received: January 7, 2014 